\title{
The effect of MHD turbulence on massive protoplanetary disk fragmentation
}

\author{
S. Fromang ${ }^{1,2}$ \\ 1 Astronomy Unit, Queen Mary, University of London, Mile End Road, London E1 4NS, UK \\ 2 Institut d'Astrophysique de Paris, 98bis Bd. Arago, 75014 Paris, France \\ e-mail: s.fromang@qmul.ac.uk
}

Received 17 March 2005 / Accepted 6 June 2005

\begin{abstract}
Massive disk fragmentation has been suggested to be one of the mechanisms leading to the formation of giant planets. While it has been heavily studied in quiescent hydrodynamic disks, the effect of MHD turbulence arising from the magnetorotational instability (MRI) has never been investigated. This paper fills this gap and presents 3D numerical simulations of the evolution of locally isothermal, massive and magnetized disks. In the absence of magnetic fields, a laminar disk fragments and clumps are formed due to the effect of self-gravity. Although they disapear in less than a dynamical timescale in the simulations because of the limited numerical resolution, various diagnostics suggest that they should survive and form giant planets in real disks. When the disk is magnetized, it becomes turbulent at the same time as gravitational instabilities develop. At intermediate resolution, no fragmentation is observed in these turbulent models, while a large number of fragments appear in the equivalent hydrodynamical runs. This is because MHD turbulence reduces the strength of the gravitational instability. As the resolution is increased, the most unstable wavelengths of the MRI are better resolved and small scale angular momentum transport starts to play a role: fragments are found to form in massive and turbulent disks in that case. All of these results indicate that there is a complicated interaction between gravitational instabilities and MHD turbulence that influences disk fragmentation processes.
\end{abstract}

Key words. accretion, accretion disks - magnetohydrodynamics (MHD) - methods: numerical stars: planetary system: formation

\section{Introduction}

The recent discovery of more than 100 extrasolar planets has renewed interest in planet formation theory. Because of selection effects, all of the planet discovered so far are planets with masses larger than the mass of Neptune. However, the problem of their formation remains unsolved, despite the attention it continues to enjoy in the recent literature (Boss 1998; Mayer et al. 2002; Rice et al. 2003). Two very different models have been proposed.

In the so-called "core accretion model", giant planets first form their cores by dust accumulation. When the core reaches a mass of the order of 10 earth masses, a phase of rapid gas accretion ensues and builds up the envelope of the planet (Pollack et al. 1996). The typical timescale to form a planet of 1 Jupiter mass in a realistic disk is of the order of $10^{7}$ years. This rather long timescale has raised questions since it is similar to the expected lifetime of the disk. However, recent studies suggest that the migration of the planet in the disk may overcome this difficulty (Hourigan \& Ward 1984; Rice \& Armitage 2003; Alibert et al. 2004).

The alternative scenario is that giant planets form directly in massive protoplanetary disks by gravitational fragmentation
(Boss 1998, 2000). Massive disks are linearly unstable to axisymmetric gravitational instabilities when the Toomre $Q$ parameter (Toomre 1964), defined by

$Q=\frac{c_{\mathrm{s}} \kappa}{\pi G \Sigma}$,

becomes smaller than 1 . In the definition above, $c_{\mathrm{s}}$ is the sound speed, $\kappa$ is the epicyclic frequency (see, e.g., Binney $\&$ Tremaine 1987), $\Sigma$ is the disk surface density and $G$ is the gravitational constant. Gaseous disks are also unstable to nonaxisymmetric perturbations when $Q \geq 1$. In the later case, spiral arms develop and transport angular momentum outward in the disk. If the nonlinear evolution of the instability is very violent, these spiral arms may fragment to form dense clumps which have been interpreted as proto-giant planets. For example, Gammie (2001) found that a disk fragments when its cooling timescale is shorter than $\tau_{\text {cool }} \sim 3 \Omega^{-1}$. This was confirmed by Rice et al. (2003) using SPH numerical simulations. Mayer et al. (2002) and Mayer et al. (2004) also found fragmentation for a variety of disk models and equations of state. However, Pickett et al. (2000) failed to obtain fragmentation in similar thermodynamic conditions. Whether or not massive disks fragment remains controversial. 
As described above, the issue of disk fragmentation has been largely addressed in the past few years. These studies omit the effect of magnetic forces, despite the known effect of even a weak magnetic field on the dynamical evolution of a disk. Indeed, rotating disks in which the angular velocity decreases outward are unstable to the MRI (Balbus \& Hawley 1991). In the last decade, numerical simulations, both local and global, have shown that its nonlinear outcome is MHD turbulence that transports angular momentum outward (see Balbus \& Hawley 1998, or Balbus 2003, for a review). Recently, Fromang et al. (2004a,b) investigated the evolution of massive, magnetized disks, in which the MRI and gravitational instabilities are expected to develop simultaneously. The main result of this study is that both instabilities are tighly coupled and strongly interact with one another, leading to a quasi-periodic variation of the mass accretion rate onto the central star. However, these results were obtained using an adiabatic equation of state, a regime in which no gravitational collapse was found in the disk. The latter is more likely to occur in isothermal disks, since the pressure support is less important in that case. The main goal of this paper is to investigate the influence of MHD turbulence on the evolution of isothermal disks, with an emphasis on the question of their fragmentation.

The plan of the paper is as follows. Section 2 presents the numerical methods used and the initial conditions of the simulations. Section 3 describes their results, focusing first on purely hydrodynamic cases and then on full MHD simulations. These results are finally discussed in Sect. 4.

\section{Method}

The relevant equations are those of ideal MHD for a selfgravitating fluid, in which $\rho$ is the mass density, $\boldsymbol{v}$ is the velocity, $\boldsymbol{B}$ is the magnetic field, $P$ is the gas pressure and $\Phi=$ $\Phi_{\mathrm{S}}+\Phi_{\mathrm{c}}$ is the total gravitational potential, which has contributions $\Phi_{\mathrm{S}}$ from the disk self-gravity and $\Phi_{\mathrm{c}}$ from a central mass:

$$
\begin{aligned}
& \frac{\partial \rho}{\partial t}+\boldsymbol{\nabla} \cdot(\rho \boldsymbol{v})=0, \\
& \rho\left(\frac{\partial \boldsymbol{v}}{\partial t}+(\boldsymbol{v} \cdot \boldsymbol{\nabla}) \boldsymbol{v}\right)=-\boldsymbol{\nabla} P-\rho \boldsymbol{\nabla} \Phi+\frac{1}{4 \pi}(\boldsymbol{\nabla} \times \boldsymbol{B}) \times \boldsymbol{B}, \\
& \frac{\partial \boldsymbol{B}}{\partial t}=\boldsymbol{\nabla} \times(\boldsymbol{v} \times \boldsymbol{B}), \\
& \nabla^{2} \Phi_{\mathrm{s}}=4 \pi G \rho .
\end{aligned}
$$

The equation of state is either adiabatic or locally isothermal. In the latter case, the temperature in the disk is a function of position, but not time:

$$
\frac{P}{\rho}=f(r, \phi, z)
$$

Here $(r, \phi, z)$ denotes the standard cylindrical coordinates that will be used throughout the paper. When the equation of state is adiabatic, the pressure is derived from the internal energy $e$ using

$$
P=(\gamma-1) e
$$

where $\gamma=5 / 3$ is the polytropic index. $e$ is calculated using the following energy equation:

$$
\rho\left(\frac{\partial}{\partial t}+\boldsymbol{v} \cdot \boldsymbol{\nabla}\right)\left(\frac{e}{\rho}\right)=-P \boldsymbol{\nabla} \cdot \boldsymbol{v},
$$

\subsection{Numerical procedure}

To solve the equations described above, I used the code GLOBAL (Hawley \& Stone 1995). It uses time-explicit Eulerian finite differences and is designed to handle standard cylindrical coordinates. The magnetic field is evolved using the combined Method of Characteristics and Constrained Transport algorithm (MOC-CT), which preserves the divergence of the magnetic field to machine accuracy at all time. In order to reduce the time of the simulations, the computational domain is reduced to $[0, \pi]$ in the azimuthal direction, as in Fromang et al. (2004b). Finally, outflow boundary conditions are applied in the radial and vertical directions, while periodic boundary conditions are applied in $\phi$.

The self-gravitating potential $\Phi_{S}$ is calculated with a Poisson solver well adapted to cylindrical geometry (Fromang et al. 2004b). The density distribution of the disk is first Fourier transformed in the azimuthal direction. For computational reasons, only those Fourier components $\rho_{m}$ whose index $m$ ranges between 0 and $m_{\max }$ are retained at this stage. For each of them, the procedure is the following: (1) from $\rho_{m}$, the gravitational potential $\Phi_{\mathrm{S}}^{m}$ is obtained at the boundary of the computational domain using an expansion in Legendre functions (Cohl \& Tohline 1999). (2) $\Phi_{\mathrm{S}}^{m}$ is calculated everywhere on the computational domain using the Successive Over Relaxation method (Hirsch 1988). Finally, $\Phi_{S}$ is reconstructed using the values of $\Phi_{\mathrm{S}}^{m}$, with $m \in\left[0, m_{\max }\right]$.

One has to understand that the value of $m_{\max }$ described above is crucial for the problem of disk fragmentation addressed in this paper. Indeed, the larger the number of coefficient included in the calculation of $\Phi_{\mathrm{S}}$, the smaller the structures that can be described by the simulation. Of course, this comes also with a larger computational time. Nevertheless, in order to accurately describe the tiny structures associated with the possible fragmentation of the disks, I used $m_{\max }=32,64$ or 128 in this work, in contrast with the lower value $m_{\max }=8$ typically used by Fromang et al. (2004b) in adiabatic simulations where only large structures appeared.

\subsection{Initial conditions}

The properties of the various runs performed are summarized in Table 1. The first column gives the label of the models. HD refers to hydrodynamic models and T refers to runs starting with an initial toroidal field. Column 2 gives the number $m_{\max }$ of Fourier coefficient included in the calculation of the gravitational potential. The third column gives the initial ratio $\langle\beta\rangle$ of the volume averaged thermal pressure to the volume averaged magnetic pressure. Column 4 gives the number of grid points $\left(N_{r}, N_{\phi}, N_{z}\right)$ of the run and Col. 5 describes the equation of state ("iso" refers to a locally isothermal equation of state). 
Table 1. The first column gives the model label. The second and third columns respectively give the number $m_{\max }$ of Fourier coefficient included in the calculation of the gravitational potential and $\langle\beta\rangle$, the initial ratio of thermal to magnetic pressure. The resolution $\left(N_{r}, N_{\phi}, N_{z}\right)$ of the model is shown in Col. 4, while Col. 5 gives the equation of state used ("iso" stands for locally isothermal and "adia" for an adiabatic equation of state). Finally, the last column indicates whether or not fragments were found in the isothermal runs.

\begin{tabular}{lccccc}
\hline \hline Model & $m_{\max }$ & $\langle\beta\rangle$ & Resolution & EQS & Fragments? \\
\hline HDISO2 & 32 & $+\infty$ & $(128,64,128)$ & iso & No \\
HDISO3 & 64 & $+\infty$ & $(128,128,128)$ & iso & Yes \\
HDISO4 & 128 & $+\infty$ & $(128,256,64)$ & iso & Yes \\
\hline T2 $^{*}$ & 0 & 8 & $(128,64,128)$ & adia & No \\
T3 $^{*}$ & 0 & 8 & $(128,128,128)$ & adia & No \\
T4$^{*}$ & 0 & 8 & $(128,256,64)$ & adia & No \\
TISO2 & 32 & 8 & $(128,64,128)$ & iso & No \\
TISO3 & 64 & 8 & $(128,128,128)$ & iso & No \\
TISO4 & 128 & 8 & $(128,256,64)$ & iso & Yes \\
\hline
\end{tabular}

Whether or not fragments form in the disk is described in the last column.

Some care has to be taken regarding the initial conditions of each of these models. Model T2* was already presented in Fromang et al. (2004b): assuming an adiabatic equation of state, it calculates the evolution of a disk initially in equilibrium, with a mass half that of the central mass, threaded by a toroidal weak magnetic field for which the ratio of the volume averaged thermal pressure to the volume averaged magnetic pressure $\langle\beta\rangle$ is initially 8 . The MRI grows because of the magnetic field, and the disk eventually becomes fully turbulent. No gravitational instability develops since $m_{\max }=0$ in this model, which is evolved for about 6 orbits at the location of the initial outer edge of the disk. At this stage, it has reached a quasi-stationary turbulent state typical of the outcome of global simulations of zero mass disks: mass slowly falls onto the central object under the action of the Maxwell and the Reynolds stresses, while angular momentum is transported in the outer parts of the disks, which spreads as a consequence. At the same time, a tenuous, magnetized corona builds up above and below the disk main body.

This quasi-stationary state emerging from model $\mathrm{T} 2 *$ after 6 orbits was used as the initial condition for model TISO2. To do so, the temperature was calculated at the end of model T2* everywhere on the grid and then kept constant for the further evolution of model TISO2, whose equation of state is locally isothermal.

In order to compare model TISO2 with an hydrodynamic model whose properties are as closely matched as possible, the disk structure resulting from model T2* was also used to compute the initial conditions for model HDISO2. The procedure used to do so is as follows: the result of model T2* was first azimuthally averaged and the magnetic fields were removed. A small random perturbation was then applied to the disk before restarting the simulation with a locally isothermal equation of


Fig. 1. Snapshots of the logarithm of the equatorial density of the disk for model HD2, respectively at times $t=9.0,9.2,9.3,9.5,9.9$ and 10.3 from top left to bottom right. The structure of the disk varies very rapidely. Short lived high density clumps are visible in each snapshots.

state. Note that this sudden removal of the relatively weak magnetic field does not strongly affect the disk global structure.

The other simulations were initialized in a similar way. Model T3*, TISO3, HDISO3 and T4*, TISO4, HDISO4 are the high resolution equivalent to model T2*, TISO2, HDISO2. For models T4*, TISO4, and HDISO4, in which 256 grid cells were used in the azimuthal direction, the number of cells in the vertical direction was decreased by a factor of two. This is to save computational time. Even with this reduction of $N_{z}$, model TISO4 required more than $1100 \mathrm{~h}$ of CPU time per orbit (measured at the initial outer edge of thi disk) on a Pentium $3.06 \mathrm{GHz}$ Xeon chip. This is mostly because of the large number of Fourier components used in that case $\left(m_{\max }=\right.$ 128 in both HDISO4 and TISO4).

\section{Results}

\subsection{Hydrodynamic simulations}

\subsubsection{Disk fragmentation}

I first present the results of the fiducial hydrodynamic model HDISO3, for which $N_{\phi}=128$. After about one orbit, a strong gravitational instability develops in the disk as a consequence of its large mass. The early evolution of the instability is similar to what was obtained using an adiabatic equation of state (Fromang et al. 2004b). However, the spiral arms that form in the present case are stronger because of the weaker pressure support. When the gravitational instability saturates, the disk fragments into dense clumps. This can be seen in Fig. 1, which shows six snapshots of the logarithm of the density in the equatorial plane of the disk at times (measured in the orbital time at the initial outer edge of the disk) 9.0, 9.2, 9.3, 9.5, 9.9 and 10.3 (from top left to bottom right). Note that the results of the simulation have been extended by symmetry to cover the range $[0,2 \pi]$ in this figure, which explains why an even numbers of clumps is always seen. Figure 1 clearly illustrates the fact that the state of the disk is changing very rapidly. The clumps form, their density increases, reaches a maximum and starts to decline. They eventually completely dissolve in the background flow in less than the dynamical 

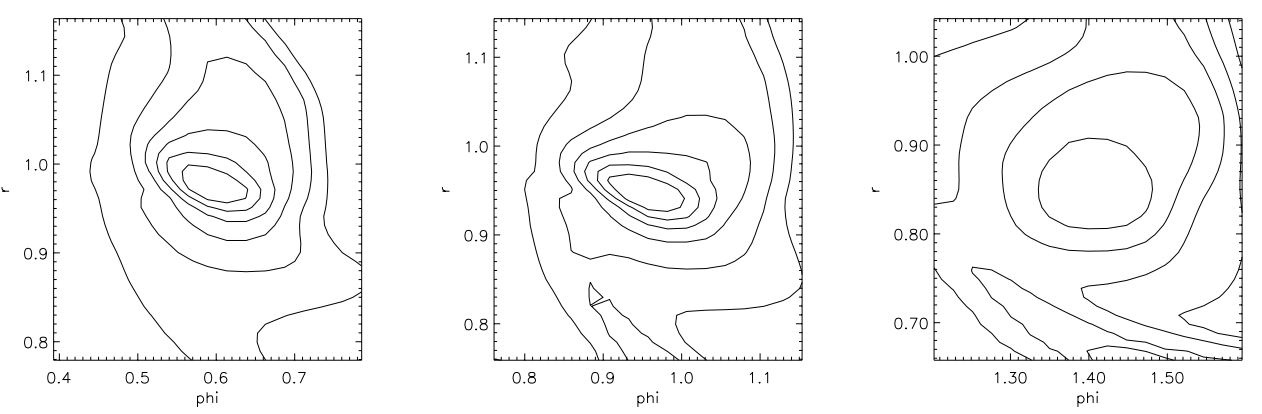

Fig. 2. Detailed structure of the clump shown in the bottom right frame of Fig. 1 at times $t=10.21,10.29$ and 10.37 . $\rho_{\max }$ is respectively 93 , 70 and 3.6 at these times. Six contours levels are represented in each panels. They correspond to the density values: $\log \rho=\log \rho_{\max }-$ $0.5, \log \rho_{\max }-1$, etc.

Table 2. Properties of the four clumps visible in the bottom row of Fig. 1 (model HDISO3). The first line of the table shows the corresponding time at which each of these properties are calculated. It serves as a way of labelling the clumps. The following lines then give the orbital radius $a$ of the clump, its peak density $\rho_{\max }$, its mass $M_{10}$ (resp $M_{100}$ ), ie the mass contained in the volume where the density is larger than $\rho_{\max } / 10\left(\operatorname{resp} \rho_{\max } / 100\right)$, normalized by the total mass of the disk $M_{\mathrm{T}}$, the Jeans mass $M_{\mathrm{J}}$, the radius $r_{\text {clump }}$ of the clump, its Hill radius and finally the ratio $\alpha$ between its thermal and gravitational energy.

\begin{tabular}{lcccc}
\hline \hline$t$ (orbits) & 9.5 & $9.9-1$ & $9.9-2$ & 10.3 \\
\hline$a$ & 0.69 & 0.52 & 0.65 & 0.97 \\
$\rho_{\max }$ & 106 & 357 & 256 & 134 \\
$M_{10} / M_{\mathrm{T}}$ & $3.7 \times 10^{-3}$ & $2.8 \times 10^{-3}$ & $4.4 \times 10^{-3}$ & $2.4 \times 10^{-3}$ \\
$M_{100} / M_{\mathrm{T}}$ & $8.6 \times 10^{-3}$ & $5.8 \times 10^{-3}$ & $7.0 \times 10^{-3}$ & $4.1 \times 10^{-3}$ \\
$M_{\mathrm{J}} / M_{\mathrm{T}}$ & $6.5 \times 10^{-4}$ & $4.3 \times 10^{-4}$ & $4.4 \times 10^{-4}$ & $6.2 \times 10^{-5}$ \\
$r_{\text {clump }}$ & $2 \times 10^{-2}$ & - & $2 \times 10^{-2}$ & $2 \times 10^{-2}$ \\
$r_{\mathrm{H}}$ & $8.3 \times 10^{-2}$ & $5.7 \times 10^{-2}$ & $8.3 \times 10^{-2}$ & 0.1 \\
$\alpha$ & 0.33 & 0.5 & 0.28 & 0.11 \\
\hline
\end{tabular}

timescale. They barely complete more than a quarter of their orbit before disappearing.

Table 2 presents a list of the properties of the fragments at times 9.5, 9.9 ( 2 clumps exist in the disk at that time and are labelled 9.9-1 and 9.9-2) and 10.3, when 3D outputs of all the variables were saved. The first and second lines show the radius $a$ at which each clump has formed and its maximum density $\rho_{\max }$. The mass $M_{10}$ and $M_{100}$, normalized by the total disk mass $M_{\mathrm{T}}$ are given by the third and forth lines. They respectively correspond to the mass of the gas contained in the volume where $\rho>\rho_{\max } / 10$ and $\rho>\rho_{\max } / 100$. This should be compared with the local Jeans mass, given by the fifth line. Line 6 and 7 respectively give the radius of the clump $r_{\text {clump }}$ and its Hill radius $r_{\mathrm{H}}$, defined by

$r_{\mathrm{H}}=a\left(\frac{M_{10}}{3 M_{\mathrm{c}}}\right)$,

where $M_{\mathrm{c}}=1$ is the central point mass. Finally, the ratio $\alpha$ between the thermal and the gravitational energy of the clump is given by the last line.
For each cases, the maximum density is above 100 (the maximum density in the initial disk model is 1). The mass of the clumps normalized by the total disk mass is a few times $10^{-3}$. Since the latter is of the order of the central point mass, it means that the fragments have masses of the order of the mass of Jupiter (for a solar-type central mass). The following lines of Table 2 give some insights into the stability properties of the clumps: their mass is about one order of magnitude larger than the local Jeans mass. Their radius is smaller than the Hill radius: they are not destroyed by tidal effects. Finally, $\alpha$ is smaller than one, showing that they are gravitationally bound.

All these parameters, taken together, indicates that the fragments should not disappear. But still, they dissolved very rapidly in the surrounding disk. This is detailed in Fig. 2: for the clump that formed around $t=10.3$ (bottom right panel of Fig. 1), density contours in the vicinity of the fragment are represented. The maximum of the density in the equatorial plane of the disk decreases from 93 to 3.6 between $t=10.21$ and $t=10.37$, during which the fragment performed less than one fifth of its orbit. The reason for this disappearance, most likely numerical, is discussed in Sect. 4.1

\subsubsection{Numerical effects}

Given the difficulties in accurately describing fragmentation in protoplanetary disks, it is important to investigate the numerical artifacts that could influence the outcome of the simulation presented above. The most important of such effects is obviously numerical resolution, especially in the azimuthal direction. In model HDISO2, $N_{\phi}$ equals 64 , while in model HDISO4, $N_{\phi}=256$.

The qualitative evolution of model HDISO4 is very similar to that of model HDISO3: fragments form rapidly after the gravitational instability saturates. The maximum density they reach is larger $\left(\rho_{\max } \sim 500\right)$, but, like model HDISO3, they disappear very quickly. For the low resolution run HDISO2, however, no fragment appears at all in the disk. This trend shows how sensitive the issue of resolution is on disk fragmentation. Indeed, this qualitative difference arises even though the development of the instability seems to be very similar for all models. This can been seen in Fig. 3, which shows the time history 


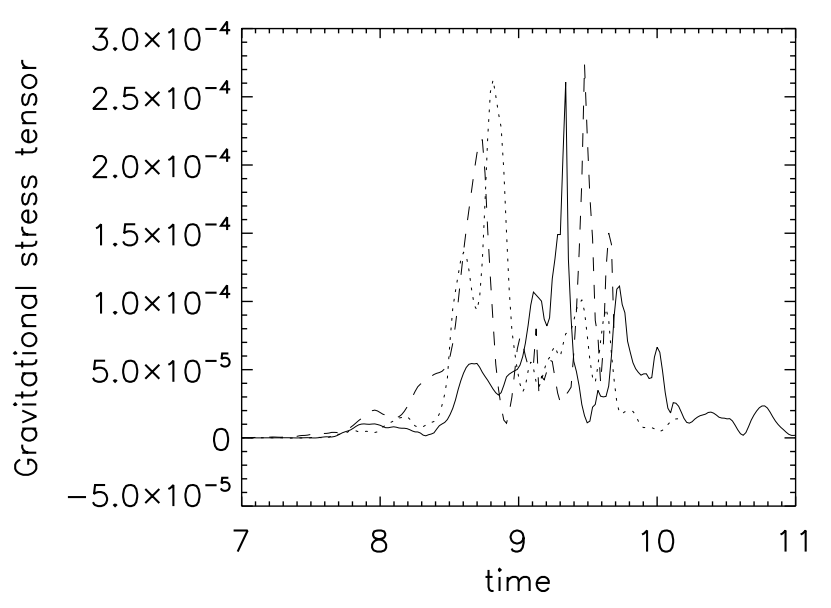

Fig. 3. Time evolution of the volume averaged gravitational stress tensor for model HDISO2 (dotted line), HDISO3 (solid line) and HDISO4 (dashed line). The peak value of $\left\langle T_{r \phi}^{\text {grav }}\right\rangle(t)$ is comparable in all models even though their resolution in the azimuthal direction is different.



Fig. 4. Logarithm of the density in the equatorial plane of a turbulent disk (model TISO3) at time $t=6.3$, when the density reaches its largest value $\rho_{\max }=16$.

of the volume averaged gravitational stress tensor. It is defined as (Fromang et al. 2004b):

$\left\langle T_{r \phi}^{\text {grav }}\right\rangle(t)=\frac{1}{4 \pi G} \int_{V} \frac{\partial \Phi_{\mathrm{s}}}{\partial r} \frac{\partial \Phi_{\mathrm{s}}}{\partial \theta} \mathrm{d} r \mathrm{~d} \theta \mathrm{d} z$.

The time history of $\left\langle T_{r \phi}^{\text {grav }}\right\rangle$ is shown in Fig. 3 for model HDISO2 (dotted line), HDISO3 (solid line) and HDISO4 (dashed line). For each cases, it starts to rise after about 8.5 orbits, and reaches similar peak values of $2.5 \times 10^{-4}$. The curves are all very similar, showing that the azimuthal resolution has little effect on the strength of the instability itself, despite the qualitative differences in the fragmentation of the disk.

\subsection{MHD simulations}

In this section, I compare the results of model TISO3 and TISO 4 with their hydrodynamic counterpart HDISO3 and HDISO4. The results of model TISO2, for which $N_{\phi}=64$, are very similar to those of model TISO3 and will not be described further.

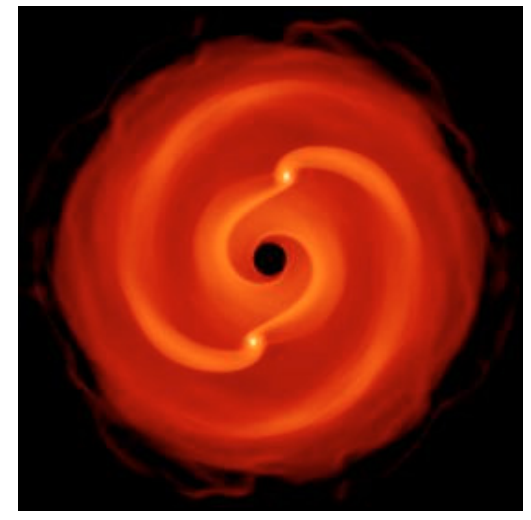

Fig. 5. Same as Fig. 4, but for model TISO4 at time $t=6.2$. The largest value of the density in this case is $\rho_{\max }=550$.

In both TISO3 and TISO4, a gravitational instability quickly develops on top of MHD turbulence. However, the qualitative evolution of the instability is dramatically different in both models. In the lower resolution case TISO3, no disk fragmentation is observed, while fragments quickly form in the high resolution model TISO4. This is illustrated by Figs. 4 and 5. The former shows the logarithm of the density in the midplane of the disk for model TISO3 after 6.2 orbits, which corresponds to the time when it reaches its largest density, $\rho_{\max } \sim 16$. Note how different the disk structure looks compared to its hydrodynamic equivalent HDISO3 (Fig. 1). On the contrary, Fig. 5 clearly shows that fragments formed in model TISO4, despite the presence of MHD turbulence. The properties and time history of the clumps are very similar to those observed in the hydrodynamic models described above. The likely origin of the difference between models TISO3 and TISO4 is discussed in the following section.

\section{Discussion}

\subsection{The Jeans length}

A critical parameter to take into account in the simulations presented above is the Jeans length, defined by:

$\lambda_{\mathrm{J}}=\left(\frac{\pi c_{\mathrm{s}}^{2}}{G \rho}\right)^{1 / 2}$

Truelove et al. (1997) showed that spurious fragmentation can occur if the Jeans length is not well resolved at all times during the disk evolution and that the largest grid cell on the computational domain should be about 4 times larger than $\lambda_{\mathrm{J}}$. In isothermal simulations, it is very difficult to resolve the Jeans length during the disk fragmentation: since the sound speed is constant, $\lambda_{\mathrm{J}}$ increases as $(1 / \rho)^{1 / 2}$. It is therefore important to follow its behaviour. At each radii, one can calculate the minimum value of $\lambda_{\mathrm{J}}$ in the equatorial plane. Its radial profile is represented in Fig. 6 for model HDISO3 at times $t=0$ (dotted-dashed line) and $t=9.9$ (solid line). The dashed line represents the largest grid size as a function of $r$ and the dotted line shows 4 times this value. At $t=0$, it is obvious that the Jeans length is well resolved and satisfies the Truelove 


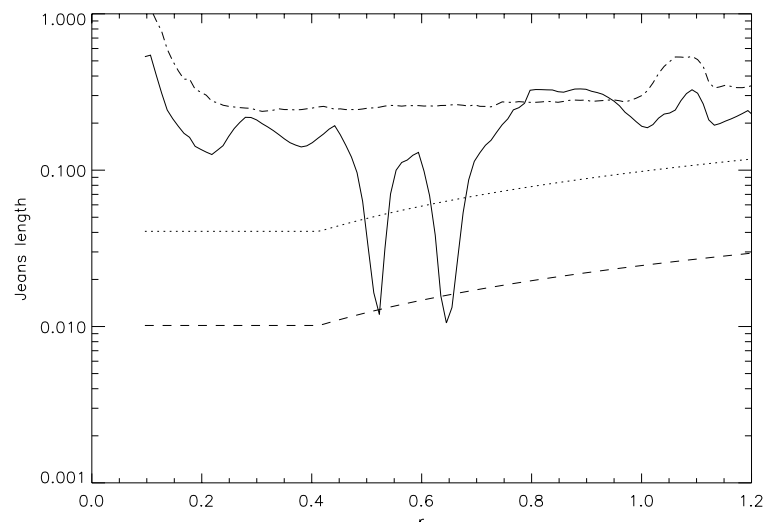

Fig. 6. Jeans length vs radius for model HDIOS3 at time $t=0$ (dotteddashed line) and $t=9.9$ (solid line). The dashed line shows the maximum of the radial and azimuthal spacing of the grid as a function of radius. The dotted line plots four times this value.

criterion. However, at $t=9.9$, two fragments formed in the disk and the resolution of the simulation becomes coarser than the local Jeans length. According to the Truelove criterion, the evolution of the fragments after this point is not meaningful and should not be regarded as being physical. On top of this, the size of the forming clumps themselves at this stage of the simulation is of the order of a few grid cells. The gravitational potential they create (and which governs their subsequent collapse) is not accurately evaluated at this scale because of the finite resolution of the grid. Their later evolution in the simulation is not well described. Both of these arguments show that these calculations can only be used as a diagnostic for disk fragmentation.

As a conclusion, the hydrodynamic simulations tell us that massive quiescent disk should fragment in the conditions studied here. However, they cannot tell us anything about the long term evolution of the clumps that formed, even though their stability properties, presented above, suggest that they may survive.

Figure 7 is similar to Fig. 6: it compares the grid resolution with the local Jeans length for the MHD turbulent disk models TISO3 (dotted-dashed line) and TISO4 (solid line) respectively at times $t=6.2$ and $t=6.3$ (i.e. at the same time as the snapshots shown in Figs. 4 and 5). In the case of model TISO3, the minimum value of the local Jeans length (which decreases at the location of the spiral arms), is roughly three times the resolution of the simulation. The Truelove criterion is only marginally violated in that case. However, in similar conditions, clumps are seen to form in model HDISO3, which shows that MHD turbulence clearly has an effect in that case. Figure 7 also shows that the case of model TISO4 is very similar to the hydrodynamic models for which fragmentation is found: the local Jeans length decreases as the density of the fragments increases, until the former becomes smaller than the resolution. The subsequent evolution of the clump is not accurately described by the simulations.

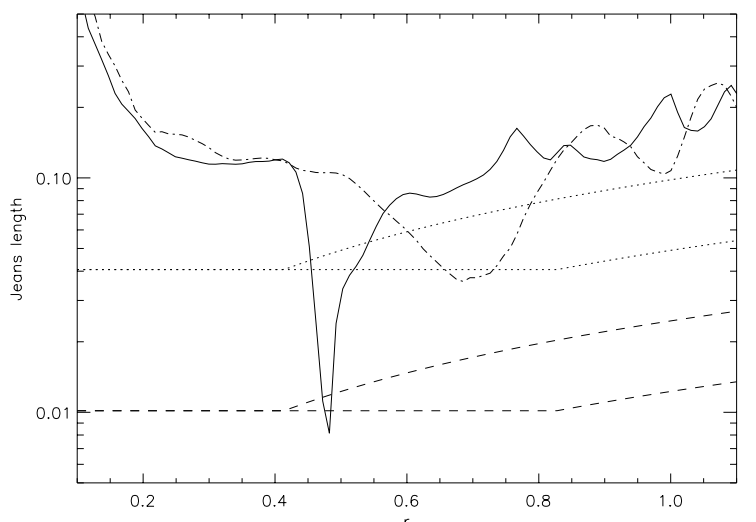

Fig. 7. Same as Fig. 6, but for models TISO3 (dotted-dashed line) at time 6.3 and TISO 4 at time 6.2. The dotted and dashed lines have the same meaning as for Fig. 6 (the upper curves show the case of model TISO3, for which $N_{\phi}=128$, while the lower curves correspond to model TISO4).



Fig. 8. Time history of the volume averaged gravitational stress tensor for models HDISO3 (solid line), TISO3 (dotted line) and TISO4 (dashed line). The earlier growth of the gravitational instability in the MHD cases is triggered by the large density perturbations due to MHD turbulence.

\subsection{The effect of MHD turbulence}

The results of model TISO3 and TISO4, together with those of their hydrodynamic counterpart HDISO3 and HDISO4 suggest that MHD turbulence affects the outcome of gravitational instabilities in disks. Although the precise interplay between these processes is difficult to understand given the complexity of the problem and its sensitivity to numerical resolution, some indications are provided by a detailed analysis of the simulations. Figure 8 shows the time history of the volume averaged gravitational stress tensor $\left\langle T_{r \phi}^{\text {grav }}\right\rangle$ (defined in Sect. 3.1.2) for models HDISO3 (solid line), TISO3 (dotted line) and TISO4 (dashed line). The maximum value reached by $\left\langle T_{r \phi}^{\text {grav }}\right\rangle$ is smaller when the disk is turbulent, suggesting that the strength of the gravitational instability itself is reduced by the presence of the turbulence. It may explain why no fragments form in model TISO3, as the gravitational stress is decreased by roughly a factor of two compared to model HDISO3 
(Note that, even though the evolution of the stress is shown for a rather short period of time, the difference between the various runs is meaningful because this is precisely during that time that fragments forms or not). This effect of MHD turbulence on the strength of the gravitational instability was already noted in simulations using an adiabatic equation of state (Fromang et al. 2004b). In adiabatic simulations, this decrease of the gravitational stress did not affect the qualitative outcome of the gravitational instability. However, in model TISO3 it prevents the density to become large enough in the spiral arm to trigger their collapse.

Figure 8 also shows that $\left\langle T_{r \phi}^{\text {grav }}\right\rangle$ is larger in model TISO4 (in which clumps form) than in TISO3. This is not due to the gravitational instability alone, since no such dependency is observed in the hydrodynamic models HDISO2, HDISO3 and HDISO4. Nor is it due to the MHD turbulence alone, since its properties are similar in model TISO3 and TISO4. However, as is explained below, the disk fragmentation (as well as the increase of the gravitational stress tensor) observed in model TISO4 could be due to the small scale angular transport properties of the MRI. In cases, such as the present one, when the magnetic field is mostly toroidal, the most unstable mode of the MRI has an azimuthal wavenumber $m_{\text {crit }}$ that satisfies the relation (Balbus \& Hawley 1998)

$\frac{m_{\mathrm{crit}}}{R} v_{A} \sim \Omega$,

which gives

$m_{\text {crit }} \sim \frac{R \Omega}{c_{\mathrm{s}}} \sqrt{\frac{\beta}{2}}$.

At the position where the fragment forms in model TISO4 (see Fig. 5), the properties of the disk model indicate that $R \Omega / c_{\mathrm{s}} \sim$ 16 , while the turbulence is characterized by $\beta \sim 10$. These values gives $m_{\text {crit }} \sim 35$. Such a mode is barely resolved in model TISO3 (only 7 grid cells per wavelength) but much better described in model TISO4 which has twice the resolution. In the latter case, it grows on an orbital timescale and helps to remove the angular momentum of the collapsing body. In the former case, the growth rate of this small scale mode is lower and it is likely it will be less important in the collapse process.

To summarize, these results suggests that, on top of the Jeans length, it is important in calculations of the present type to well resolve the length $\lambda_{B}$ defined by

$\lambda_{B}=v_{\mathrm{a}} T_{\text {orb }}$

where $T_{\text {orb }}$ is the orbital period in the disk.

Finally, it is interesting to compare the present results to those of Kim et al. (2003) who performed local calculations of giant molecular cloud formation in the presence of MHD turbulence. They found that density fluctuations triggered by the MRI can be swing-amplified and lead to disk fragmentation. This is not observed here. The reason for this difference lie in the initial setup of both calculations: Kim et al. (2003) start their simulations with vertical fields with a non-zero net flux that produces a stronger turbulence than in the present work. Indeed, the ratio of the total stress to the pressure they obtain is $\sim 0.2$, while it is an order of magnitude smaller here
(Fromang et al. 2004b). The larger density fluctuations that result are more likely to be gravitationnally bound in their case than in the present one.

\section{Conclusion}

This paper presents a collection of hydrodynamic and magnetohydrodynamic 3D numerical simulations of the evolution of massive isothermal disks.

In hydrodynamic simulations, the disk is found to fragment into high density clumps when the number of grid cells $N_{\phi}$ is larger than 128 in the azimuthal direction. The fragments, whose mass is comparable to the mass of Jupiter, disappear very quickly in the disk. However, due to the limited resolution of the simulations, the local Jeans length is no longer resolved when their density has increased by about two orders of magnitudes. Moreover, at these small scales, the collapse of the clumps themselves is not accurately described because the gravitational potential is smoothed at the grid scale. This is probably what causes their quick disappearance. At the very least, the long term behavior of these fragments cannot be studied with these simulations, although various criteria suggest that they could survive for an extended period of time.

When a weak magnetic field is present initially in the disk, it becomes turbulent because of the nonlinear development of the MRI. This turbulent nature of the flow has an effect on the evolution of the gravitational instability: no fragment is found when $N_{\phi}=128$, in contrast with the hydrodynamic model having the same resolution. However, fragments appear when the azimuthal resolution is increased to $N_{\phi}=256$. An analysis of these results suggest that they are a consequence of two competing effects: on the one hand, MHD turbulence tends to decrease the strength of the gravitational instability, as was already noted in a previous study (Fromang et al. 2004b): this is sufficient to suppress gravitational fragmentation in the lower resolution case. On the other hand, when the most unstable modes of the MRI are resolved, small scale angular momentum transport appears to help the formation of bound fragments. This interplay between disk fragmentation and radial transfer of angular momentum within an overdense region was already suggested by Kim et al. (2003).

Despite the difficulties related to the limited numerical resolutions of the simulations, the results presented in this paper indicate that MHD turbulence is an important component in the process of massive disk fragmentation. Future studies are needed to quantify the importance of the turbulence and to investigate whether the fragments that form are truly long lived or quickly dissolve in the background flow. To cope with the large resolutions needed to adress this problem, adaptative mesh refinement techniques, possibly in cylindrical coordinates, may prove to be the method of choice.

Acknowledgements. The simulations presented in this paper were performed at the Institut du Développement et des Ressources en Informatique Scientifique and at Queen Mary University of London. The author acknowledges C. Terquem and S. Balbus whose comments on an earlier draft of this paper greatly improved its contents. 


\section{References}

Alibert, Y., Mordasini, C., \& Benz, W. 2004, A\&A, 417, L25

Balbus, S., \& Hawley, J. 1991, ApJ, 376, 214

Balbus, S., \& Hawley, J. 1998, Rev. Mod. Phys., 70, 1

Balbus, S. A. 2003, ARA\&A, 41, 555

Binney, J., \& Tremaine, S. 1987, Galactic dynamics (Princeton University Press)

Boss, A. P. 1998, ApJ, 503, 923

Boss, A. P. 2000, ApJ, 536, L101

Cohl, H. S., \& Tohline, J. E. 1999, ApJ, 527, 86

Fromang, S., Balbus, S. A., \& De Villiers, J. 2004a, ApJ, 616, 357

Fromang, S., Balbus, S. A., Terquem, C., \& De Villiers, J. 2004b, ApJ, 616, 364

Gammie, C. F. 2001, ApJ, 553, 174
Hawley, J., \& Stone, J. 1995, Comput. Phys. Commun., 89, 127

Hirsch, C. 1988, Numerical Computation of Internal and External Flows - vol. 1, Fundamentals of Numerical Discretization (Wiley) Hourigan, K., \& Ward, W. R. 1984, Icarus, 60, 29

Kim, W., Ostriker, E. C., \& Stone, J. M. 2003, ApJ, 599, 1157

Mayer, L., Quinn, T., Wadsley, J., \& Stadel, J. 2002, Science, 298, 1756

Mayer, L., Quinn, T., Wadsley, J., \& Stadel, J. 2004, ApJ, 609, 1045

Pickett, B., Durisen, R., Cassen, P., \& Mejia, A. 2000, ApJ, 540, L95

Pollack, J., Hubickyj, O., Bodenheimer, P., et al. 1996, Icarus, 124, 62

Rice, W., Armitage, P., Bonnell, I., et al. 2003, MNRAS, 339, 1025

Rice, W. K. M., \& Armitage, P. J. 2003, ApJ, 598, L55

Toomre, A. 1964, ApJ, 139, 1217

Truelove, J. K., Klein, R. I., McKee, C. F., et al. 1997, ApJ, 489, L179 